\title{
A Novel Hybrid Approach for Optimal Reactive Power Dispatch under Unbalanced Conditions
}

\author{
Rohan Anil Nikam \\ Department of Mechanical \& Industrial Engineering \\ University of Illinois at Chicago, USA \\ rohananilnikam.01@gmail.com
}

\begin{abstract}
Recently, the Optimal Reactive Power Dispatch (ORPD) is gaining a considerable attention in the power systems, as they decide the security as well as economy of a power system. The major target behind this works is to upgrade the power system efficiency by means of optimally allocating the real power, so that the power loss should be minimized, and voltage profiles should be enhanced. With this intention, here and optimization algorithm referred as "Trial based Update on Whale and Particle swarm Algorithm (TU-WPA)" is implied, which an amalgamation of two algorithms is called, "Particle Swarm Optimization (PSO) and Whale Optimization Algorithm (WOA)". The TU-WPA model fine tunes the key parameters like "load reactance; voltage and transformer tap settings". Finally, the performance of the TU-WPA is compared over the existing works under "IEEE 14 and the IEEE 39 bus systems". On observing the best performance of the adopted scheme, a higher value $(0.35327)$ is recorded, while the compared models attains comparatively lower values, i.e. $\mathrm{GA}=0.26496, \mathrm{FF}=0.27496, \mathrm{PSO}=0.256, \mathrm{ABC}=0.3296, \mathrm{WOA}=0.20333, \mathrm{DA}=0.29432$ and $\mathrm{CS}=0.30496$.
\end{abstract}

Keywords: Optimal Reactive Power Dispatch; Active Power Loss Minimization; Voltage Profile Improvement; Inequality And Equality Constraints; TU-WPA

\begin{tabular}{ll} 
Nomenclature & \\
\hline Abbreviations & Descriptions \\
\hline AP \& RP & Active And Reactive Power \\
AP loss & Active Power Loss \\
ADMM & Alternating Direction Method Of Multipliers \\
ABC & Artificial Bee Colony \\
APL & Active Power Regulation \\
CS & Cuckoo Search \\
DERs & Distributed Energy Resources \\
DE & Differential Evolution \\
EFFA & Enhanced Firefly Algorithm \\
FF & Firefly Algorithm \\
GA & Genetic Algorithm \\
HMPSO & Hybrid Multi-Swarm Particle Swarm Optimisation \\
IPM & Interior Point Method \\
LP & Linear Programming \\
M-OOP & Multi-Objective Optimisation Problem \\
MORPD & Multi-Objective Optimal Reactive Power Dispatch \\
MODE & Multi-Objective Differential Evolution \\
OOAR-PDP & Objective Optimal Active And Reactive Power Dispatch Problems \\
ORPF & Optimal Reactive Power Flow \\
ORPD & Optimal Reactive Power Dispatch \\
OPF & Optimal Power Flow \\
PSO & Particle Swarm Optimization \\
QP & Quadratic Programming \\
QODE & Quasi-Oppositional Differential Evolution \\
QOBL & Quasi-Oppositional Based Learning \\
RPD & Reactive Power Dispatch \\
\hline
\end{tabular}




\begin{tabular}{ll} 
TC & Transfer Conductance \\
TU-WPA & Trial Based Update On Whale And Particle Swarm Algorithm \\
TLs & Transmission Line \\
VS & Voltage Stability \\
VD & Voltage Deviation \\
WOA & Whale Optimization Algorithm \\
\hline
\end{tabular}

\section{Introduction}

The Power firm of a country shapes the fundamental national foundation of that particular country's economy as it is the entrepreneur and innovatively escalated industry. In mid 80's the interest for power was low and thus little power stations were accessible in the area [6] [7]. The current day power framework is huge with numerous producing stations, as it has more popularity for farming, business and local shoppers. In these power advancements, the significant issues are the power framework strength [8] [22] [23]. The fundamental factor causing voltage unsteadiness is the failure of the power framework in fulfilling the responsive power need at intensely focused on frameworks, and this keeps it from keeping up the ideal voltages [9] [10] [11]. The receptive power/voltage control limits, responsive power repaying gadgets just as the heap qualities are the elements contributing the voltage breakdown. It is basic to streamline the receptive power dispatch so as to keep up the voltage dependability, power framework misfortunes and to control the activities of the influence framework [12] [13] [33].

The APL and the RPD are the two significant imperatives for the practical activity of a power framework [14] [15]. The expense of creation of RP is regularly low, yet it presents colossal downsides on the active power transmission misfortune just as active power production. In the TLs, the active power stream as far as the transmission just as dispersion voltage should be controlled with responsive power [20] [21]. The OPF is proficient in deciding the ideal settings for not many of the factors concerning the power framework control so as to decide the target capacities in regards to the framework boundaries [16][34] [35]. the main sub problems of OPF is optimal reactive power dispatch (ORPD) problem which includes generator output voltages, compensators, tap ratios of transformers, and outputs of shunt reactive sources. This sub problem is used to minimize interested objective functions such as transmission losses while satisfying a given set of operating and physical limitations. The whole ORPD problem is considered as a non-linear multi-modal optimization problem with a combination of discrete and continuous variables. In addition, the sub-issue of OPF is the ORPF and its significant target is to reduce the "AP loss and maximization of voltage profile and stability real power loss" with the guide of influence framework control factors changes like "AP loss and maximization of voltage profile and stability". In the customary methodologies, a few exemplary improvement procedures like LP, QP and IPM were utilized to supersede the issues identified with advancement of the receptive power dispatch [17] [18] [19]. In any case, they flopped in accomplishing the worldwide minima and experienced unpredictability. Therefore, it is basic to have an ideal method, to defeat these difficulties. Moreover, the deployment of the optimization algorithm [25] [26] [36] will be a solution for most of the optimizations problems. The major contribution of this paper is as follows:

- Enhances the efficiency of power system by optimizing the real power allocation parameters such as load reactance; voltage and transformer tap settings using a new metaheuristic algorithm.

- Introduces Trial based Update on Whale and Particle swarm Algorithm, which is an amalgamation of two algorithms namely PSO and WOA.

The leftover sections of this paper are organized as: Section II reviews the recent works undergone relating the current research work. Section III tells about the optimal RP dispatch under unbalanced conditions: Objective function. Section IV depicts the TU-WPA algorithm for optical RP dispatch: Solution encoding. The resultants acquired with the TU-WPA are discussed in Section V. Finally, a strong conclusion is provided to the current research work in Section VII.

\section{Literature Review}

\subsection{Related Works}

In 2015, Srivastava and Singh [1] have developed a HMPSO algorithm for RP dispatch issues solving. The HMPSO was the amalgamation of the DE and the PSO. "The RPD problem is formulated as nonlinear, constrained M-OOP with equality and inequality constraints for minimisation of power losses and improvement of voltage profile simultaneously". The proposed model was tested on "standard IEEE 30 bus and practical 75-bus Indian systems". 
In 2015, Liang et al. [2] have proposed EFFA with the intention of solving the power dispatch problems in terms of "AP \& RP" in the wind generation unit. the authors have considered the RP as well as the active power's optimal dispatches at the same time. the losses in the TLs as well as the VDs in the load buses were reduced by the RP dispatch, which takes into consideration the parameters like "load tap changer positions of transformers, the RP injection of capacitors, and the voltages of slack bus and PV buses". They have utilized the "IEEE 30-bus system" in order to test the presented work in terms of OOAR-PDP.

In 2016, Basu [3] has suggested MODE for MORPD. this was achieved by means of lessening the "active power transmission loss and VD and maximizing VS". All these were accomplished by varying control variables of the power system like the "terminal voltages, transformer taps and RP output of shunt VAR compensators". the presented work was tested for the achievement of the objective by means of implementi8ng it on "IEEE 30-bus, 57-bus and 118-bus systems".

In 2015, Robbins and Garcia [4] have proposed a novel approach in DERs for regulating the voltage of the bus. They have formulated an OPF problem for radial power systems, by means of introducing a branch power flow modeling approach. in OPF, certain non-linear terms were leveraged and hence a convex QP was formed. In addition, they have developed a distributed algorithm based on the ADMM to solve the issues related to $\mathrm{QP}$.

In 2014, Zhou et al. [5] have proffered quasi-oppositional differential evolution approach in a power system in order to solve the issues related to the RP dispatch problem. This QODE have utilized the QOBL in order to initialize the population. Further, with the proposed model, the control variables settings were found in terms of "generator terminal voltages, transformer tap settings and RP output of shunt VAR compensators". Thus, the presented work had gained the "minimum AP loss, improved voltage profile and enhanced VS". The presented work was tested on "IEEE 30-bus, 57-bus and 118-bus test systems".

In 2020, Ettappan et al. [33] have proposed the ORPD for real power loss minimization, voltage deviation minimization and voltage stability enhancement using $\mathrm{ABC}$ Algorithm. The $\mathrm{ABC}$ algorithm was used to find the setting of control variables such as generator voltage magnitude, tap position of tap changing transformer and reactive power output of the compensation devices. The proposed algorithm was tested on IEEE 30 and 57 bus systems, Simulation results shown that the proposed approach converges to better solutions and much faster than the earlier reported approaches in the literature.

\section{Proposed ORPD Model}

\subsection{ORPD Model Under Unbalanced Condition}

Under the unbalanced condition, the achievement of ORPD is highly challenging. Thus, this research work goes towards the objective of AP loss minimization as well as stability and voltage profile improvement in the unbalanced condition of the network. The dependent variables in the vector form are expressed in Eq. (1).

$$
\mathrm{Z}=\left[\mathrm{Po}_{\mathrm{G} 1}, \mathrm{Vo}_{1-1} \ldots \ldots . \mathrm{Vo}_{1-\mathrm{NPQ}}, \mathrm{Q}_{\mathrm{G}-1} \ldots . . . \mathrm{Q}_{\mathrm{G}-\mathrm{NG}}\right]
$$

Here, the voltage bus $\mathrm{PQ}(\mathrm{i}=1,2 \ldots . \mathrm{NPQ})$ is denoted as $\mathrm{Vo}_{\mathrm{li}}$ and the slack bus power is indicated using the notation $\mathrm{Po}_{\mathrm{G}}$. The real power from the generator $(\mathrm{i}=1,2 \ldots . \mathrm{NG})$ is denoted as $\mathrm{Q}_{\mathrm{Gi}}$. Here, the count of buses in the generator is denoted as $N G$. In addition, the available count of the $\mathrm{PQ}$ bus is symbolized as NPQ . The control variable vector is given by Eq. (2). In the voltage controlled bus, the terminal voltage is represented as $\mathrm{Vo}_{\mathrm{Gi}}$. Then, the overall number of shunt VAR compensator's is NC, and NT is the count of quantity of the tap clanging transformer's.

$$
\mathrm{U}=\left[\mathrm{Vo}_{\mathrm{G}-1}, \ldots . . \mathrm{Vo}_{\mathrm{G}-\mathrm{NG}}, \mathrm{Q}_{\mathrm{C}-1} \ldots . . \mathrm{Q}_{\mathrm{C}-\mathrm{NC}}, \mathrm{t}_{1}, \ldots . . \mathrm{t}_{\mathrm{NT}}\right]
$$

Active Power Load Minimization: The equality as well as the inequality factors is generated by these selected parameters. The objective function of the current research work is manifested in Eq. (3).

$$
\operatorname{Obj}=\alpha \mathrm{F}_{\mathrm{a}}+(1-\alpha) \mathrm{F}_{\mathrm{b}}
$$

Here, $F_{a}$ indicates the AP loss and $F_{b}$ signifies the VD. The mathematical formula for AP loss minimization is mathematically expressed in Eq. (4).

$$
\mathrm{F}_{\mathrm{a}}=\mathrm{Po}_{1}=\sum_{\mathrm{k}=1}^{\mathrm{N}} \mathrm{g}_{\mathrm{k}}\left[\mathrm{Vo}_{\mathrm{m}}^{2}+\mathrm{Vo}_{\mathrm{n}}^{2}-2 \mathrm{Vo}_{\mathrm{m}} \mathrm{Vo}_{\mathrm{n}} \cos \left(\delta_{\mathrm{m}}-\delta_{\mathrm{n}}\right)\right]
$$


The AP loss of the system is denoted as $\mathrm{Po}_{1}$. The count of TLss is depicted as $\mathrm{N}$. Then, among the buses $\mathrm{m}^{\text {th }}$ and $\mathrm{n}^{\text {th }}$, the $\mathrm{k}^{\text {th }}$ branch conductance is denoted as $\mathrm{g}_{\mathrm{k}}$. For the buses, $\mathrm{m}^{\text {th }}$ and $\mathrm{n}^{\text {th }}$, the voltage phase angles are ${ }^{\delta_{m}}$ and $\delta_{n}$, correspondingly.

VD: The voltage magnitude $\left(V_{i}\right)$ reduction at varied loads of a bus system, from a predetermined reference value $\left(\mathrm{V}^{\text {ref }}\right)$ is utilized with the aim of enhancing the voltage profile. The improvement in voltage profile is mathematically exhibited in Eq. (5).

$$
\mathrm{F}_{\mathrm{b}}=\mathrm{Vo}_{\mathrm{D}}=\sum_{\mathrm{i}=1}^{\mathrm{LB}} \mathrm{Po}_{\mathrm{f}} \psi\left(\mathrm{Vo}^{\min }-\mathrm{Vo}_{\mathrm{m}}\right)+\mathrm{Po}_{\mathrm{f}} \psi\left(\mathrm{Vo}_{\mathrm{m}}-\mathrm{Vo}^{\max }\right)
$$

The step function $\psi(\mathrm{z})$ is expressed in Eq. (6)

$$
\psi(\mathrm{x})=\left\{\begin{array}{ccc}
1 ; & \text { if } & \mathrm{z} \geq 0 \\
0 ; & \text { otherwise } &
\end{array}\right.
$$

The count of load buses LB in Eq. (7) can be given as

$$
\left|\mathrm{Vo}_{\mathrm{m}}\right|^{2}=\left|\mathrm{Vo}_{\mathrm{i}}\right|^{2}-2\left(\tilde{\mathrm{r}}_{\mathrm{im}} \mathrm{Po}_{\mathrm{im}}+\tilde{\mathrm{z}}_{\mathrm{im}} \mathrm{Q}_{\mathrm{im}}\right)+\mathrm{c}_{\mathrm{im}}(, \mathrm{Q})
$$

The susceptance $\mathrm{x}$ of the line is mathematically shown in Eq. (8)

$$
\tilde{\mathrm{r}}_{\mathrm{im}}=\operatorname{Re}\left\{a \mathrm{a}^{\mathrm{H}}\right\} \otimes \mathrm{r}_{\mathrm{im}}+\operatorname{Im}\left\{\mathrm{aa}^{\mathrm{H}}\right\} \otimes \mathrm{x}_{\mathrm{im}}
$$

The resistance $r$ of the line is shown in Eq. (9)

$$
\begin{aligned}
& \tilde{\mathrm{x}}_{\mathrm{im}}=\operatorname{Re}\left\{\mathrm{aa}^{\mathrm{H}}\right\} \otimes \mathrm{x}_{\mathrm{im}}-\operatorname{Im}\left\{\mathrm{aa}^{\mathrm{H}}\right\} \otimes \mathrm{r}_{\mathrm{im}} \\
& \mathrm{a}=\left[\begin{array}{lll}
1 & \left.\mathrm{e}^{-\mathrm{j} 2 \pi / 3} \mathrm{e}^{\mathrm{j} 2 \pi / 3}\right]
\end{array}\right] \\
& \mathrm{c}_{\mathrm{im}}=\left[\mathrm{z}_{\mathrm{im}}\left[\mathrm{S}_{\mathrm{im}}^{*} / / \mathrm{Vo}_{\mathrm{i}}^{*}\right]\right] \otimes\left[\mathrm{z}_{\mathrm{im}}^{*}\left[\mathrm{~S}_{\mathrm{im}_{0}} \cdot / \mathrm{Vo}_{\mathrm{i}}\right]\right] \\
& \mathrm{z}_{\mathrm{im}}=\mathrm{r}+\mathrm{jx} \\
& \mathrm{S}_{\mathrm{im}}=\left[\mathrm{Po}_{\mathrm{im}}+\mathrm{jQ} \mathrm{Q}_{\mathrm{im}}\right] \otimes\left[\tilde{\mathrm{z}}_{\mathrm{im}}\left(\mathrm{Po}_{\mathrm{im}}-\mathrm{jQ} \mathrm{Q}_{\mathrm{im}}\right)\right] \\
& \tilde{\mathrm{z}}_{\mathrm{im}}=\mathrm{z}_{\mathrm{im}} \otimes\left(\mathrm{a}_{\mathrm{i}} \mathrm{a}_{\mathrm{i}}^{\mathrm{H}}\right)
\end{aligned}
$$

"It is the necessity of an every buses in the power system to withstand the voltage that is below the normal operating conditions and these buses need to adapt themselves to the disturbance like change in load and system configuration. At present most of the major networks deteriorate due to the instability in voltage". The VS is achieved by means of lessening the VS indicator. At every bus, the L -index value $\left(L_{n}\right)$ specified the voltage condition that is distorted of that specific bus. Mathematically, the $L_{n}$ of $n^{\text {th }}$ bus is denoted as per Eq. (15), where $\mathrm{n}=1,2 \ldots \ldots ., \mathrm{NPQ}$. In addition here the PV bus count is represented as NPV . The sub-matrices of YBUS is denoted as $\mathrm{Y}_{b}$ and $\mathrm{Y}_{\mathrm{a}}$. Then, the constraints of $\mathrm{PQ}$ and PV bus are separated for YBUS and is expressed mathematically in Eq. (17).

$$
\begin{aligned}
& \mathrm{L}_{\mathrm{n}}=\left|1-\sum_{\mathrm{m}=1}^{\mathrm{NPV}} \mathrm{F}_{\mathrm{nm}} \frac{\mathrm{Vo}_{\mathrm{m}}}{\mathrm{Vo}_{\mathrm{n}}}\right| \\
& \mathrm{F}_{\mathrm{nm}}=\left[\mathrm{Y}_{\mathrm{a}}\right]^{-1}\left[\mathrm{Y}_{\mathrm{b}}\right] \\
& {\left[\begin{array}{c}
\mathrm{I}_{\mathrm{PQ}} \\
\mathrm{I}_{\mathrm{PV}}
\end{array}\right]=\left[\begin{array}{c}
\mathrm{Y}_{\mathrm{a}} \mathrm{Y}_{\mathrm{b}} \\
\mathrm{Y}_{\mathrm{c}} \mathrm{Y}_{\mathrm{d}}
\end{array}\right]\left[\begin{array}{c}
\mathrm{V}_{\mathrm{PQ}} \\
\mathrm{V}_{\mathrm{PV}}
\end{array}\right]}
\end{aligned}
$$

Then, for the entire PQbuses, the $\mathrm{L}$ - Index value is expressed. Here, the value of $\mathrm{L}_{\mathrm{n}}$ is fixed as 0 or 1 on the basis of the $\mathrm{n}^{\text {th }}$ bus VD as well as no load stipulations. Therefore, the objective function could be given by Eq. (18), where $\mathrm{L}_{\mathrm{n}}=1,2 \ldots . . \mathrm{NPQ}$.

$$
\mathrm{F}_{\mathrm{c}}=\max \left(\mathrm{L}_{\mathrm{n}}\right)
$$

\subsection{Inequality and Equality Constraints}

"The inequality constraints are-non binding constraints and there is no necessity for the real power output to stay within its maximum limit". The power system is controlled by the equality factors, which encloses the load flow formulations. Mathematically, these load flow formulations are expressed in Eq. (19) and (20), respectively, where $\mathrm{m}=1,2, \cdots, \mathrm{NB}$. The count of buses is represented as NB . the generation 
of AP \& RPs in $\mathrm{m}^{\text {th }}$ bus is denoted as $\mathrm{P}_{\mathrm{Gm}}$ and $\mathrm{Q}_{\mathrm{Gm}}$, respectively. With respect to demand, the AP \& RPs of $\mathrm{m}^{\text {th }}$ bus is specified as $\mathrm{P}_{\mathrm{Dm}}$ and $\mathrm{Q}_{\mathrm{Dm}}$, respectively.

$$
\begin{aligned}
& \mathrm{Po}_{\mathrm{Gm}_{m}}-\mathrm{Po}_{\mathrm{Dm}}-\mathrm{Vo}_{\mathrm{m}} \sum_{\mathrm{n}=1}^{\mathrm{NB}} \mathrm{Vo}_{\mathrm{n}}\left[\mathrm{B}_{\mathrm{mm}} \sin \left(\delta_{\mathrm{m}}-\delta_{\mathrm{n}}\right)+\mathrm{G}_{\mathrm{mn}} \cos \left(\delta_{\mathrm{m}}-\delta_{\mathrm{n}}\right)\right]=0 \\
& \mathrm{Q}_{\mathrm{Gm}}-\mathrm{Q}_{\mathrm{Dm}}-\mathrm{Vo}_{\mathrm{m}} \sum_{\mathrm{n}=1}^{\mathrm{NB}} \mathrm{Vo}_{\mathrm{n}}\left[\mathrm{B}_{\mathrm{mn}} \sin \left(\delta_{\mathrm{m}}-\delta_{\mathrm{n}}\right)+\mathrm{G}_{\mathrm{mn}} \cos \left(\delta_{\mathrm{m}}-\delta_{\mathrm{n}}\right)\right]=0
\end{aligned}
$$

In addition, the among the $\mathrm{m}^{\text {th }}$ bus and $\mathrm{n}^{\text {th }}$ bus, the $\mathrm{TC}$ is denoted as $\mathrm{B}_{\mathrm{mn}}$. The magnitude of the output voltage as well as the generator RP need to be maintained within the limits during the designing of a power system. Eq. (21) represents the mathematical formula for the upper limit.

$$
\mathrm{Vo}_{\mathrm{Gm}}^{\min } \leq \mathrm{Vo}_{\mathrm{Gm}} \leq \mathrm{Vo}_{\mathrm{Gm}}^{\max }, \mathrm{m}=1,2, \ldots, \mathrm{NG}
$$

In addition, the lower limit of output voltage of generator is expressed in Eq. (22).

$$
\mathrm{Q}_{\mathrm{Gm}}^{\min } \leq \mathrm{Q}_{\mathrm{Gm}} \leq \mathrm{Q}_{\mathrm{Gm}}^{\max }, \mathrm{m}=1,2, \ldots, \mathrm{NG}
$$

In shunt VAR compensators, the real power outputs limits (lower and upper) are made and it is based on Eq. (23).

$$
\mathrm{Q}_{\mathrm{Cm}}^{\min } \leq \mathrm{Q}_{\mathrm{Cm}} \leq \mathrm{Q}_{\mathrm{Cm}}^{\max }, \mathrm{m}=1,2, \ldots, \mathrm{NC}
$$

In case of the transformer tap settings, the physical considerations exhibit the upper and lower limit values. This is expressed as per Eq. (24).

$$
\mathrm{T}_{\mathrm{m}}^{\min } \leq \mathrm{T}_{\mathrm{m}} \leq \mathrm{T}_{\mathrm{m}}^{\max }, \mathrm{m}=1,2, \ldots, \mathrm{NT}
$$

in PQ buses, the security constrain are based on the voltage magnitude and TLs loadings. The concerned limit flow of each line for the voltage of the buses is shown in Eq. (25) and Eq. (26), respectively.

$$
\begin{aligned}
& \mathrm{Vo}_{\mathrm{Im}}^{\min } \leq \mathrm{Vo}_{\mathrm{Im}} \leq \mathrm{Vo}_{\mathrm{Im}}^{\max }, \mathrm{m}=1,2, \ldots, \mathrm{NPQ} \\
& \mathrm{H}_{\mathrm{im}} \leq \mathrm{H}_{\mathrm{im}}^{\max }, \mathrm{m}=1,2, \ldots, \mathrm{N}
\end{aligned}
$$

\section{TU-WPA Algorithm for ORPD Problem : Solution Encoding}

\subsection{Solution Encoding}

To solve the problem of ORPD, the TU-WPA optimization Algorithm is introduced. The TU-WPA algorithm takes the input as real power ( Q), voltage magnitude (Vo) and transformer tap setting ( T).

\subsection{TU-WPA model}

WOA has taken as one amongst the renowned "nature-inspired meta-heuristic optimization algorithm, which follows the social behavior of the humpback whales". The standard WOA [31] is good in solving complex and realistic optimization issues. But, it suffers from lower convergence. Typically, PSO [29] is considered as a problem-solving and swarm-based foraging optimization algorithm in which the individuals are defined as particles. On the other hand, the PSO algorithm has higher convergence, yet here the local optimal issues are higher. Thus, the TU-WPA [24] algorithm is introduced here, which is a hybrid algorithm that combines the working principle of PSO and WOA.

The procedure of TU-WPA algorithm is as follows:

Step 1: The search agents of PSO and WOA are initialized as $\mathrm{X}_{\mathrm{e}}$.

Step 2: Then, the ffitness is calculated for whole search agents. Here, $\mathrm{X}_{\mathrm{e}}^{*}=$ optimal search agent.The vector position of best solution is denoted as $\mathrm{X}^{*}$.

Step 3: while $t r<\max t r$. Here ${ }^{t r}$ denotes the present generation.

Step 4: Then, for whole search agent, Update $a_{e}, A_{e} r_{e}$ and $p_{e}$. The vector coefficients are determined as $\mathrm{A}_{\mathrm{e}}$ and $\mathrm{C}_{\mathrm{e}}$, which is computed as per the Eq. (27) and (28).

$$
\begin{aligned}
& \mathrm{A}_{\mathrm{e}}=2 \mathrm{a}_{\mathrm{e}} \cdot \mathrm{r}_{\mathrm{e}}-\mathrm{a}_{\mathrm{e}} \\
& \mathrm{C}_{\mathrm{e}}=2 \cdot \mathrm{r}_{\mathrm{e}}
\end{aligned}
$$

In the respective equations, $a_{e}$ value get minimized sequentially from 2 to 0 , and random vector $r_{e}$ is enclosed within the interval $[0,1]$. Then, $\operatorname{tr}=0$ is set.

Step 5: if $(\operatorname{tr}<20)$ and if $\left(\mathrm{p}_{\mathrm{e}}<0.5\right)$ and if $\left|\mathrm{A}_{\mathrm{e}}\right|<1$, then update the agent location by Eq. (30). 


$$
\begin{aligned}
& \mathrm{D}_{\mathrm{e}}=\left|\mathrm{C}_{\mathrm{e}} \cdot \mathrm{X}_{\mathrm{e}}^{*}(\mathrm{tr})-\mathrm{X}_{\mathrm{e}}(\mathrm{tr})\right| \\
& \mathrm{X}_{\mathrm{e}}(\mathrm{tr}+1)=\mathrm{X}_{\mathrm{e}}^{*}(\mathrm{tr})-\mathrm{A}_{\mathrm{e}}-\mathrm{D}_{\mathrm{e}}
\end{aligned}
$$

Step 6: if $\left|A_{e}\right| \geq 1$, then pick up an arbitrary search agent $X_{\text {earb }}$ and update the agent location by Eq. (31). Here $\mathrm{X}_{\text {earb }}$ indicates the randomly selected value from the current population.

$$
\mathrm{X}_{\mathrm{e}}(\mathrm{tr}+1)=\mathrm{X}_{\text {earb }}-\mathrm{D}_{\mathrm{e}} \cdot \mathrm{A}_{\mathrm{e}}
$$

Generally, the whales do random search with respect to the location of other whale. thus, $\mathrm{A}_{\mathrm{e}}>1$ or $\mathrm{A}_{\mathrm{e}}<-1$ is used to skip the whales that are far away from them.

Step 7: if $\left(p_{e} \geq 0.5\right)$, then agent location of current search agent is updated by Eq. (33). This expression is the spiral equation is arithmetically defined for the distance among location of prey and humpback whale.

$$
\begin{aligned}
& \mathrm{D}_{\mathrm{e}}{ }^{\prime}=\left|\mathrm{X}_{\mathrm{e}}^{*}(\mathrm{tr})-\mathrm{X}_{\mathrm{e}}(\mathrm{tr})\right| \\
& \mathrm{X}_{\mathrm{e}}(\mathrm{tr}+1)=\mathrm{D}_{\mathrm{e}}{ }^{\prime} \cdot \mathrm{e}^{\mathrm{df}} \cdot \cos (2 \pi \mathrm{f})+\mathrm{X}_{\mathrm{e}}^{*}(\mathrm{tr})
\end{aligned}
$$

In this, logarithmic spiral shape is defined as $d$ and is termed to be constant, the uniform distribution of random number $f$ falls within the range $[-1,1]$.

Step 8: if $(\operatorname{tr}>20)$, then update the location on the basis of PSO update in Eq. (7.13)and set $\operatorname{tr}=0$.

Step 9: Check, whether any of the search agent has gone beyond the limit of the search space. Then, calculate fitness for whole search agents. Further, Update $\mathrm{X}_{\mathrm{e}}^{*}$ when other optimal solution subsists. Further, increase $\operatorname{tr}=\operatorname{tr}+1$

Step 10: Terminate

\section{Results and Discussions}

\subsection{Simulation Procedure}

The proposed ORPD model with the aid of the optimization algorithm was implemented in MATLAB, and the outcomes are observed on "IEEE 14 and the IEEE 39 standard bus systems". Moreover, the performance of the TU-WPA model was distinguished with four well-known optimization models namely, GA [27], FF [28], PSO [29], ABC [30], WOA [31], DA [32] and CS [33]. This evaluation is made in term of statistical evaluation."As the entire schemes were stochastic in nature a, respectively and they were greatly based on the initial arbitrary solutions, the arithmetical examination was made by carrying out tests for almost five times".

\subsection{Statistical Evaluation on IEEE 14 Bus System}

The TU-WPA approach is compared over the extant models and the resultant acquired are shown graphically in Fig.1. From the resultants it is much clear that the TU-WPA has attaining better ORPD, while compared over the existing ones. On observing the best performance of the adopted scheme, a higher value (0.35327) is recorded, while compared over to the other models $\mathrm{GA}=0.26496, \mathrm{FF}=0.27496$, $\mathrm{PSO}=0.256, \mathrm{ABC}=0.3296, \mathrm{WOA}=0.20333, \mathrm{DA}=0.29432$ and $\mathrm{CS}=0.30496$. Then, on the other hand, the mean performance of the TU-WPA is 0.35462 , which is $17.7 \%, 14.2 \%, 29.4 \%, 25.2 \%$ and $11 \%$ much superior to the existing models like "GA, FF, PSO, ABC, WOA, DA and CS", respectively. Thus from the overall evaluation, it is clear that the TU-WPA is much superior to the existing works, while implemented under IEEE 14 bus system. 


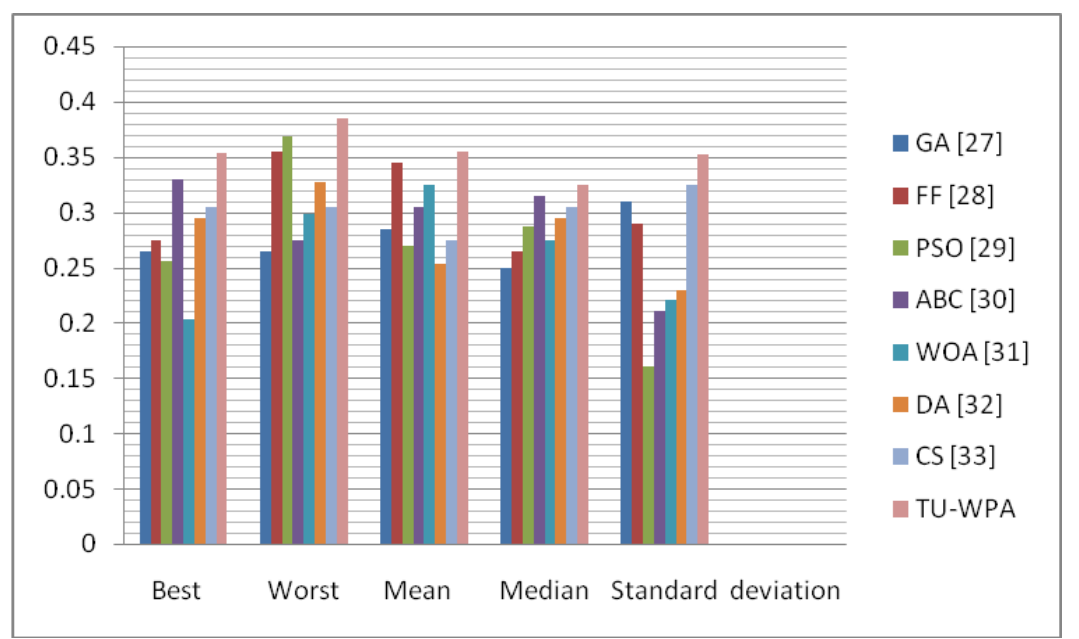

Fig. 1. Statistical analysis on TU-WPA over existing works for IEEE 14 bus system

\subsection{Statistical Evaluation on IEEE 39 Bus System}

The statistical analysis of the adopted scheme for "IEEE 39 bus system" can be obtained as shown in Fig.2. The highest best value is 38.192 and it is $48.5 \%, 45.3 \%, 40 \%, 25 \%$ and $20.6 \%$ more superior to the extant models like "GA, FF, PSO, ABC, WOA, DA and CS", respectively. The median of the TU-WPA model is 36.286, which is the highest among all other models. Thus, from the overall evaluation, it is vivid that TU-WPA model is good in solving ORPD issues.

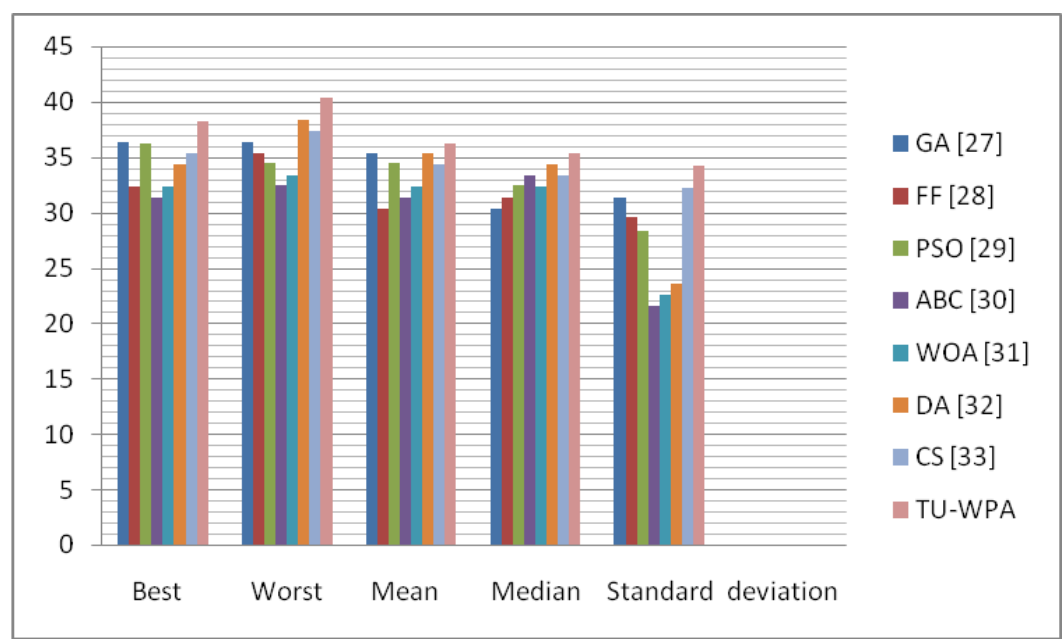

Fig. 2. Statistical analysis on TU-WPA over existing works for IEEE 39 bus system

\subsection{Convergence Analysis}

Fig.3 exhibits the convergence analysis of the TU-WPA model by varying the parameter a (fitness function). This variation in fitness is made from " $\alpha=1, \alpha=1.5, \alpha=2, \alpha=2.5, \alpha=3, \alpha=3.5$ and $\alpha=4$ ". Then for each of the variation, the change in the "loss, voltage penalty and final cost function" is computed for both the bus system. The loss function (LSI) of IEEE 14 bus is constant and it is verified from the resultant shown in Fig. 3 (a). for all variation the fitness, the loss function stays at the level of 0.9. Then, in case of voltage penalty (VSI), the TU-WPA has recorded the lowest value as 0.72 at $\alpha=2$. The final cost function s lower for the TU-WPA at in $\alpha=1$. On the other hand, the values of "loss, voltage penalty" and final cost function are measured for IEEE 39 bus systems are shown in Fig. 3 (b). The minimal loss function is recorded by the TU-WPA as 0.81 at $\alpha=4$. The final cost value is $1.8,1.5,1.29,1.27,1.27,1.54$ and 1.81 at $\alpha=1, \alpha=1.5, \alpha=2, \alpha=2.5, \alpha=3, \alpha=3.5$ and $\alpha=4$, respectively. 


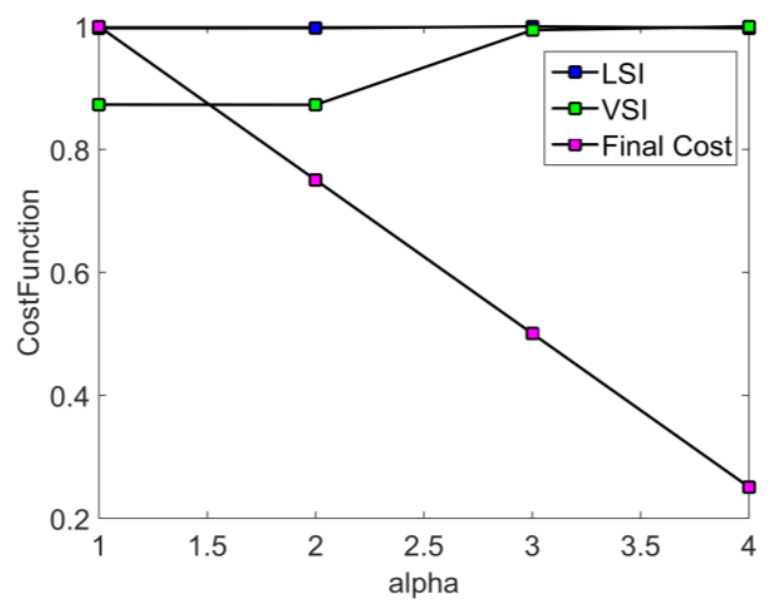

(a)

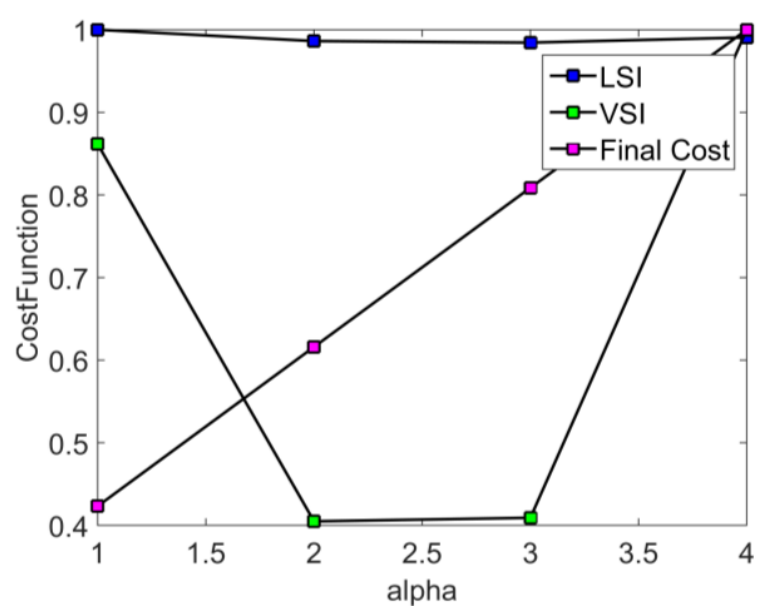

(b)

Fig. 3. Performance analysis of TU-WPA over existing models for (a) IEEE 14 bus systems and (b) IEEE 39 bus system

\section{Conclusion}

In this paper a new approach was developed for solving the issues related to the ORPD. The inequality and equality constraints were considered for solving the non-linear optimization problem and the AP loss and the VD minimization was the major objective of this research. Here, a hybrid algorithm referred as TU-WPA was used for optimization issue. The TU-WPA model's performance was verified over other existing algorithms for both the "IEEE 14 and the IEEE 39 benchmark bus systems". On observing the best performance of the adopted scheme in case IEEE 14 bus system, a higher value (0.35327) was recorded, when compared to the other models $\mathrm{GA}=0.26496, \mathrm{FF}=0.27496, \mathrm{PSO}=0.256, \mathrm{ABC}=0.3296$, $\mathrm{WOA}=0.20333, \mathrm{DA}=0.29432$ and $\mathrm{CS}=0.30496$. Thus, the betterment of the adopted work has been validated in an effective manner.

\section{Compliance with Ethical Standards}

Conflicts of interest: Authors declared that they have no conflict of interest.

Human participants: The conducted research follows the ethical standards and the authors ensured that they have not conducted any studies with human participants or animals.

\section{References}

[1] L. Srivastava and H. Singh, "Hybrid multi-swarm particle swarm optimisation based multi-objective reactive power dispatch," in IET Generation, Transmission \& Distribution, vol. 9, no. 8, pp. 727-739, may 2015.

[2] Ruey-Hsun Liang, Jia-Ching Wang, Yie-Tone Chen, Wan-Tsun Tseng,"An enhanced firefly algorithm to multiobjective optimal active/reactive power dispatch with uncertainties consideration",International Journal of Electrical Power \& Energy Systems, vol.4,pp.1088-1097,January 2015.

[3] M. Basu,"Multi-objective optimal reactive power dispatch using multi-objective differential evolution",International Journal of Electrical Power \& Energy Systems,vol.82,pp.213-224,November 2016.

[4] B. A. Robbins and A. D. Domínguez-García, "Optimal Reactive Power Dispatch for Voltage Regulation in Unbalanced Distribution Systems," in IEEE Transactions on Power Systems, vol. 31, no. 4, pp. 2903-2913, July 2016.

[5] M. Basu,"Quasi-oppositional differential evolution for optimal reactive power dispatch",International Journal of Electrical Power \& Energy Systems, Vol.78,pp.29-40,June 2016.

[6] Souhil Mouassa, Tarek Bouktir, Ahmed. Salhi, "Ant lion optimizer for solving optimal RP dispatch problem in power systems", Engineering Science and Technology, an International Journal, vol. 20, no. 3, pp. 885-895, June 2017.

[7] Pradeep Jangir, Siddharth A. Parmar, Indrajit N. Trivedi, R. H. Bhesdadiya, "A novel hybrid Particle Swarm Optimizer with multi verse optimizer for global numerical optimization and Optimal RP Dispatch problem", Engineering Science and Technology, an International Journal, vol. 20, no. 2, pp. 570-586, April 2017.

[8] Ehsan Naderi, Hossein Narimani, Mehdi Fathi, Mohammad Rasoul Narimani, "A novel fuzzy adaptive configuration of particle swarm optimization to solve large-scale optimal RP dispatch", Applied Soft Computing, vol. 53, pp. 441-456, April 2017. 
[9] Etta Grover-Silva, Robin Girard, George Kariniotakis, "Optimal sizing and placement of distribution grid connected battery systems through an SOCP optimal power flow algorithm", Applied Energy, vol. 219, pp. 385393, 1 June 2018.

[10] Abdel-Fattah Attia, Ragab A. El Sehiemy, Hany M. Hasanien, "Optimal power flow solution in power systems using a novel Sine-Cosine algorithm”, International Journal of Electrical Power \& Energy Systems, vol. 99, pp. 331-343, July 2018.

[11] Alexander Engelmann, Tillmann Mühlpfordt, Yuning Jiang, Boris Houska, Timm Faulwasser, "Distributed AC Optimal Power Flow using ALADIN", IFAC-PapersOnLine, vol. 50, no. 1, pp. 5536-5541, July 2017.

[12] Alberto Pagnetti, Mahmoud Ezzaki, Ismail Anqouda, "Impact of wind power production in a European Optimal Power Flow”, Electric Power Systems Research, vol. 152, pp. 284-294, November 2017.

[13] Partha P. Biswas, P. N. Suganthan, Gehan A. J. Amaratunga, "Optimal power flow solutions incorporating stochastic wind and solar power", Energy Conversion and Management, vol. 148, pp. 1194-1207, 15 September 2017.

[14] Erfan Mohagheghi, Aouss Gabash, Mansour Alramlawi, Pu Li, "Real-time optimal power flow with RP dispatch of wind stations using a reconciliation algorithm”, Renewable Energy, vol. 126, pp. 509-523, October 2018.

[15] Attia A. El-Fergany, Hany M. Hasanien, "Tree-seed algorithm for solving optimal power flow problem in largescale power systems incorporating validations and comparisons", Applied Soft Computing, vol. 64, pp. 307-316, March 2018

[16] Yinliang Xu, Hongbin Sun, Houde Liu, Qing Fu, "Distributed solution to DC optimal power flow with congestion management", International Journal of Electrical Power \& Energy Systems, vol. 95, pp. 73-82, February 2018.

[17] O. Aydin, S. S. Tezcan, I. Eke, M. C. Taplamacioglu, "Solving the Optimal Power Flow Quadratic Cost Functions using Vortex Search Algorithm”, IFAC-PapersOnLine, vol. 50, no. 1, pp. 239-244, July 2017.

[18] Elnaz Davoodi, Ebrahim Babaei, Behnam Mohammadi-ivatloo, "An efficient covexified SDP model for multiobjective optimal power flow”, International Journal of Electrical Power \& Energy Systems, vol. 102, pp. 254264, November 2018.

[19] C. Shilaja, K. Ravi, "Optimal Power Flow Using Hybrid DA-APSO Algorithm in Renewable Energy Resources", Energy Procedia, vol. 117, pp. 1085-1092, June 2017.

[20] Ernest Benedito, Dunstano del Puerto-Flores, Arnau Dòria-Cerezo, Jacquelien M. A. Scherpen, "Optimal Power Flow for resistive DC Networks: a Port-Hamiltonian approach”, IFAC-PapersOnLine, vol. 50, no. 1, pp. 25-30, July 2017.

[21] Vincent Roberge, Mohammed Tarbouchi, Francis Okou, "Optimal power flow based on parallel metaheuristics for graphics processing units", Electric Power Systems Research, vol. 140, pp. 344-353, November 2016.

[22] Alexander Hermann, Qiuwei Wu, Shaojun Huang, Arne Hejde Nielsen, "Convex Relaxation of Optimal Power Flow in Distribution Feeders with Embedded Solar Power”, Energy Procedia, vol. 100, pp. 43-49, November 2016.

[23] Line Roald, Maria Vrakopoulou, Frauke Oldewurtel, Göran Andersson, "Risk-based optimal power flow with probabilistic guarantees", International Journal of Electrical Power \& Energy Systems, vol. 72, pp. 66-74, November 2015

[24] G. K. Shailaja \& C. V. Guru Rao,"Robust and lossless data privacy preservation: optimal key based data sanitization",Evolutionary Intelligence, 2019

[25] Rajakumar B R, "Optimization using lion algorithm: a biological inspiration from lion's social behavior", Evolutionary Intelligence, Special Issue on Nature inspired algorithms for high performance computing in computer vision, Vol. 11, No. 1-2, pages 31-52, 2018, DOI: https://doi.org/10.1007/s12065-018-0168-y.

[26] B. R. Rajakumar, "Static and Adaptive Mutation Techniques for Genetic algorithm: A Systematic Comparative Analysis", International Journal of Computational Science and Engineering, Vol. 8, No. 2, pages: 180-193, 2013, DOI: 10.1504/IJCSE.2013.053087

[27] JohnMcCall, " Genetic algorithms for modelling and optimisation", Journal of Computational and Applied Mathematics, vol. 184, no. 1, pp. 205-222, 2005.

[28] IztokFister, IztokFisterJr, Xin-SheYang and JanezBrest, "A comprehensive review of firefly algorithms", Swarm and Evolutionary Computation, vol. 13, pp. 34-46, 2013.

[29] M.R.Tanweer, S.Suresh, and N.Sundararajan, " Self regulating particle swarm optimization algorithm", Information Sciences, vol. 294, pp. 182-202, 2015.

[30] D.KarabogaB.Basturk, " On the performance of artificial bee colony (ABC) algorithm", Applied Soft Computing, vol. 8, no. 1, pp. 687-697, 2008.

[31] Seyedali Mirjalili,Andrew Lewisa,"The Whale Optimization Algorithm",Advances in Engineering Software,vol.95,pp.51-67,May 2016.

[32] Seyedali Mirjalili,"Dragonfly algorithm: a new meta-heuristic optimization technique for solving single-objective, discrete, and multi-objective problems",Neural Computing and Applications, vol.27, no.4, pp 1053-1073, May 2016.

[33] M. EttappanV. VimalaS. RameshV. Thiruppathy Kesavan, "Optimal reactive power dispatch for real power loss minimization and voltage stability enhancement using Artificial Bee Colony Algorithm”, Microprocessors and Microsystems, vol.76, Article 103085, July 2020.

[34] Jian LiNi WangDao ZhouWeihao HuFrede Blaabjerg, "Optimal reactive power dispatch of permanent magnet synchronous generator-based wind farm considering levelised production cost minimisation", Renewable Energy, vol.145, pp. 1-12, January 2020. 
[35] Mohamed A. M. ShaheenHany M. HasanienAbdulaziz Alkuhayli, "A novel hybrid GWO-PSO optimization technique for optimal reactive power dispatch problem solution", Ain Shams Engineering JournalIn press, corrected proofAvailable online 6 August 2020

[36] Partha P. Biswas P.N. Suganthan R. MallipeddiGehan A.J. Amaratunga, "Optimal reactive power dispatch with uncertainties in load demand and renewable energy sources adopting scenario-based approach”, Applied Soft Computing, vol.75, pp. 616-632, February 2019. 